\title{
Nature of Symmetry Breaking at the Excitonic Insulator Transition: $\mathrm{Ta}_{2} \mathrm{NiSe}_{5}$
}

\author{
Giacomo Mazza, ${ }^{1,2,3, *, \dagger}$ Malte Rösner®, ${ }^{4, *, \$}$ Lukas Windgätter $\odot,{ }^{5}$ Simone Latini, ${ }^{5}$ Hannes Hübener, ${ }^{5}$ \\ Andrew J. Millis, ${ }^{6,7}$ Angel Rubio, ${ }^{5,6,8}$ and Antoine Georges $3,6,2,1, \S$ \\ ${ }^{1}$ Department of Quantum Matter Physics, University of Geneva, Quai Ernest-Ansermet 24, 1211 Geneva, Switzerland \\ ${ }^{2}$ CPHT, CNRS, Ecole Polytechnique, IP Paris, F-91128 Palaiseau, France \\ ${ }^{3}$ Collège de France, 11 place Marcelin Berthelot, 75005 Paris, France \\ ${ }^{4}$ Radboud University, Institute for Molecules and Materials, Heijendaalseweg 135, 6525 AJ Nijmegen, Netherlands \\ ${ }^{5}$ Max Planck Institute for the Structure and Dynamics of Matter, Luruper Chaussee 149, 22761 Hamburg, Germany \\ ${ }^{6}$ Center for Computational Quantum Physics, Flatiron Institute, New York, New York 10010, USA \\ ${ }^{7}$ Department of Physics, Columbia University, New York, New York 10027, USA \\ ${ }^{8}$ Nano-Bio Spectroscopy Group, Departamento de Física de Materiales, Universidad del País Vasco, 20018 San Sebastian, Spain
}

(Received 26 November 2019; accepted 23 April 2020; published 12 May 2020)

\begin{abstract}
$\mathrm{Ta}_{2} \mathrm{NiSe}_{5}$ is one of the most promising materials for hosting an excitonic insulator ground state. While a number of experimental observations have been interpreted in this way, the precise nature of the symmetry breaking occurring in $\mathrm{Ta}_{2} \mathrm{NiSe}_{5}$, the electronic order parameter, and a realistic microscopic description of the transition mechanism are, however, missing. By a symmetry analysis based on first-principles calculations, we uncover the discrete lattice symmetries which are broken at the transition. We identify a purely electronic order parameter of excitonic nature that breaks these discrete crystal symmetries and contributes to the experimentally observed lattice distortion from an orthorombic to a monoclinic phase. Our results provide a theoretical framework to understand and analyze the excitonic transition in $\mathrm{Ta}_{2} \mathrm{NiSe}_{5}$ and settle the fundamental questions about symmetry breaking governing the spontaneous formation of excitonic insulating phases in solid-state materials.
\end{abstract}

DOI: 10.1103/PhysRevLett.124.197601

Introduction.-Spontaneous symmetry breaking is a fundamental organizing principle for understanding the emergence of long-range order. Identifying the underlying symmetry breaking is thus a key step in the characterization of the ordered phase. This can be an elusive task when the symmetry breaking field cannot be directly tuned experimentally or when different types of ordering are coupled. The so-called excitonic insulator is a prominent example of such an elusive state of matter. This phase [1-4] has been identified with the spontaneous condensation of excitons (bound electron-hole pairs) stemming from the Coulomb attraction between electrons and holes in the conduction and valence bands. Excitonic condensation has been observed and intensively investigated in specially designed devices such as bilayer quantum Hall systems [5-9] or by photo stimulation of electron-hole pairs [10-12]. In contrast, spontaneous excitonic condensation in bulk materials still remains an open question and its detection a major challenge.

$\mathrm{Ta}_{2} \mathrm{NiSe}_{5}$ has been proposed as a candidate material hosting a homogeneous excitonic condensate [13-16], i.e., without charge or other nonzero momentum order [17-19]. $\mathrm{Ta}_{2} \mathrm{NiSe}_{5}$ undergoes a structural transition from a hightemperature orthorhombic to a low-temperature monoclinic phase at $T_{s} \simeq 328 \mathrm{~K}[13,20,21]$. Proposed evidence for excitonic condensation occurring simultaneously with the structural transition includes a characteristic flattening of the valence band close to the $\Gamma$ point $[14,15,22]$, the opening of a gap in the electronic spectrum [16,23,24], and coherent oscillations reminiscent of the excitation of an amplitude mode of the condensate [25]. Due to its characteristic chain structure $\mathrm{Ta}_{2} \mathrm{NiSe}_{5}$ has so far been interpreted as a quasi one-dimensional excitonic insulator [26,27] and Kaneko et al. [27] proposed a scenario in which the coupling of the excitonic condensate with phonons gives rise to a combined excitonic and structural instability.

The following symmetry considerations, however, call into question the very notion of excitonic condensation in the solid-state context. Condensation implies the breaking of a continuous symmetry. In the case of excitonic condensation this would be the breaking of the $U_{X}(1)$ symmetry related to the conservation of relative charge between valence and conduction states. Bulk materials, however, generally lack such a $U_{X}(1)$ symmetry due to the hybridization between conduction and valence bands. The only continuous symmetry being present is the one related to the global charge conservation $U_{N}(1)$. Nonetheless, internal discrete symmetries of the solid, such as crystal symmetries, can result in an approximate realization of the relative charge conservation with symmetry-forbidden hybridizations in particular regions of the Brillouin zone. Therefore, we propose here that the spontaneous hybridization introduced 


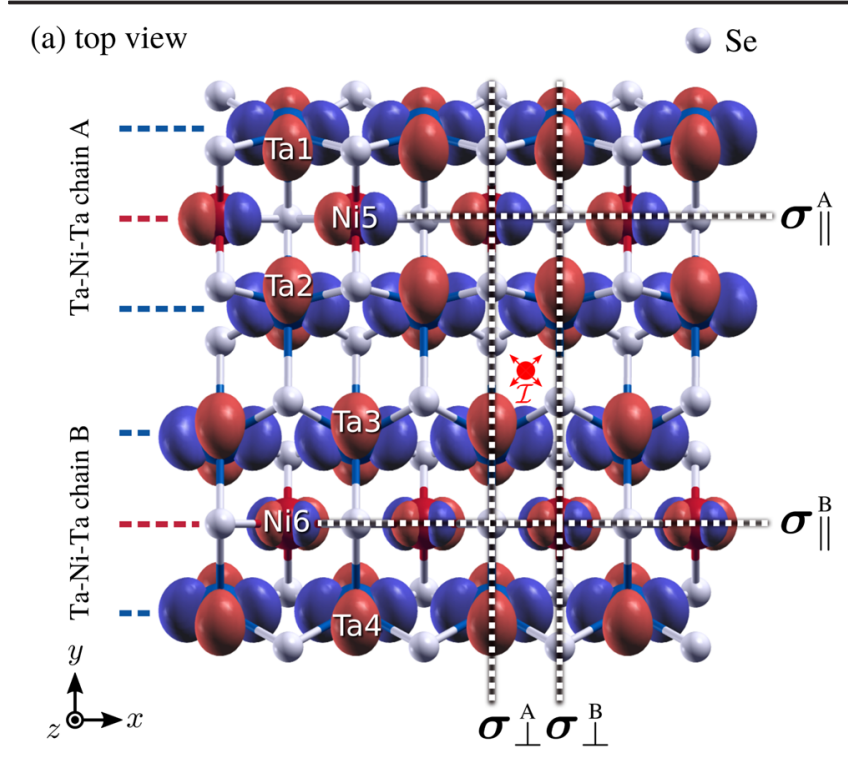

(b) side view

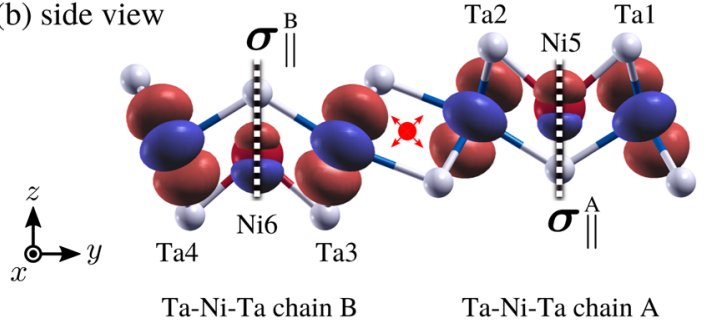

FIG. 1. Top and side view of the $\mathrm{Ta}_{2} \mathrm{NiSe}_{5}$ lattice structure including isosurfaces of Wannier wave functions localized at Ta and $\mathrm{Ni}$ positions (red and blue correspond to opposite MLFWamplitude signs). Dashed lines (red dots) indicate reflection (inversion) symmetries.

by an excitonic instability represents a general mechanism for breaking internal discrete symmetries rather than a condensation phenomenon resulting from the breaking of a continuous symmetry.

We demonstrate this concept for the case of $\mathrm{Ta}_{2} \mathrm{NiSe}_{5}$ by uncovering the symmetries that are broken by an excitonic instability. To this end, we construct a minimal yet realistic model for $\mathrm{Ta}_{2} \mathrm{NiSe}_{5}$ including its electronic band structure and electron-electron interactions from first principles. We show the existence of an electronic instability of excitonic origin leading to an electronic phase that breaks a set of discrete symmetries of the high-temperature orthorombic phase and is compatible with the low-temperature monoclinic structure. This analysis settles the fundamental question of identifying which symmetries are broken at the excitonic transition and is therefore of general relevance to the understanding and the eventual control of such transitions in $\mathrm{Ta}_{2} \mathrm{NiSe}_{5}$ and solid-state materials in general.

Crystal symmetries in $\mathrm{Ta}_{2} \mathrm{NiSe}_{5}$. - We perform DFT calculations in the high-temperature orthorhombic phase, with $a \simeq 3.51 \AA$ and $b \simeq 15.79 \AA$ being the lattice constants in $x$ and $y$ directions of the Bravais lattice [28]. This unit-cell is composed of two formula units with atoms

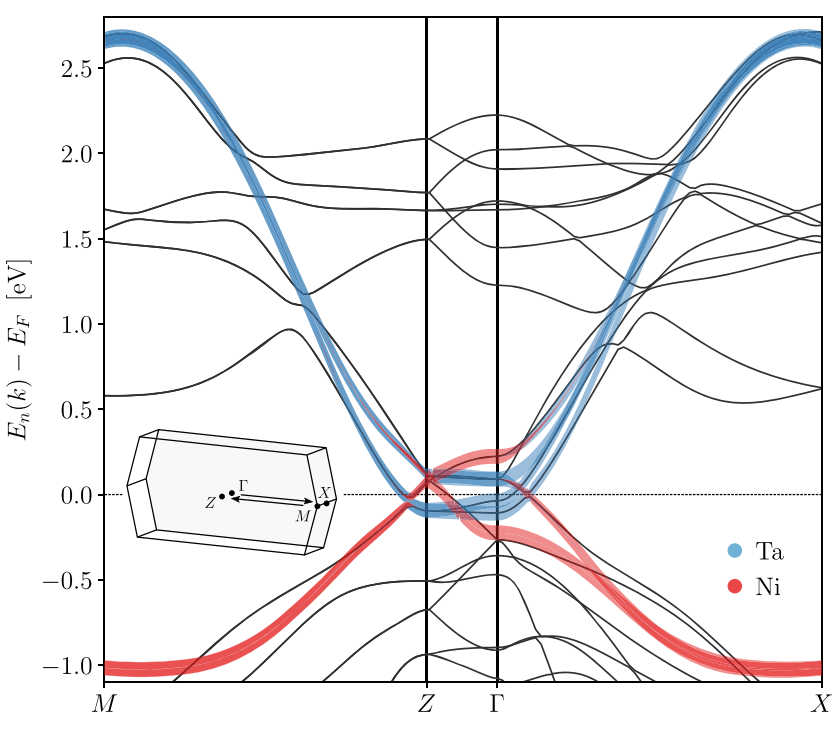

FIG. 2. Ab initio band structure (black lines) together with a fatbands representation of the Wannier model. Thick blue (red) lines represent $\mathrm{Ta}(\mathrm{Ni})$ contributions as resulting from the Wannier model.

arranged in two parallel Ta-Ni-Ta chains ( $A$ and $B$ ) along the $x$ direction. The chains are shifted by half a lattice constant along $x$ and displaced along $z$, which results in four reflection symmetries with planes parallel and perpendicular to the Ta-Ni-Ta chains $\left(\boldsymbol{\sigma}_{\| / \perp}^{A / B}\right)$ and one inversion symmetry point $\mathcal{I}$, as depicted in Fig. 1. Based on these ab initio calculations, we construct six $d_{x z}$-like maximally localized Wannier functions (MLWF) centered at the $\mathrm{Ta}$ and $\mathrm{Ni}$ positions, which are shown in Fig. 1. In each Ta-Ni-Ta chain the Ta-centered $d_{x z}$ orbitals, $\varphi_{\mathrm{Ta}}(\vec{R})$, are aligned along the chains and tilted around the $x$ axis following the Ta-Se bonds [see Fig. 1(b)]. The Ni-centered $d_{x z}$ MLWFs, $\varphi_{\mathrm{Ni}}(\vec{R})$, are also parallel to the Ta-Ni-Ta chains, but rotated by $45^{\circ}$ around the $y$ axis. The Se contributions are thus indirectly accounted for by deforming and rotating the $d_{x z}$ orbitals.

The reflection symmetries act differently on the Taand Ni-centered MLWFs. While the $\varphi_{\mathrm{Ta}}(\vec{R})$ MLWFs are unaffected by all reflections, $\varphi_{\mathrm{Ni}}(\vec{R})$ change sign under $\sigma_{\perp}$. It follows that the intrachain Ta-Ta and Ni-Ni hoppings $t_{\mathrm{TaTa} / \mathrm{NiNi}}(\vec{R})=\left\langle\varphi_{\mathrm{Ta} / \mathrm{Ni}}^{A / B}(\vec{R})|\hat{H}| \varphi_{\mathrm{Ta} / \mathrm{Ni}}^{A / B}(0)\right\rangle$ have opposite signs. We find $t(\overrightarrow{0})_{\mathrm{TaTa}} \approx-640 \mathrm{meV}$ and $t(\overrightarrow{0})_{\mathrm{NiNi}} \approx$ $250 \mathrm{meV}$, which are respectively mainly responsible for the conduction and valence bands dispersions in the $\overline{\mathrm{MZ}}$ and $\overline{\Gamma X}$ directions of the Brillouin zone. These bands are about 2.5 and $1.5 \mathrm{eV}$ wide with predominant $\mathrm{Ta}$ (blue) and $\mathrm{Ni}$ (red) character as visible in the Wannier-interpolated band structure in Fig. 2. Conduction and valence bands overlap along $\overline{Z \Gamma}$ where the bands become much less dispersive and are mainly characterized by bonding and antibonding splittings of the Ta- and Ni-states between the two Ta-Ni-Ta chains. 
Importantly, hopping matrix elements between Ta- and Ni-like states within the chains are not forbidden by any symmetry. In fact, even in the orthorhombic phase we obtain non-zero matrix elements between $\mathrm{Ta}-$ and $\mathrm{Ni}$ MLWFs within the same chain $t_{\mathrm{TaNi}}(\vec{R})$. Specifically, $t_{\mathrm{TaNi}}(\overrightarrow{0}) \approx 36 \mathrm{meV}$ which decreases with the distance $R_{x}$ along the chains. This result is universal to all tested DFT exchange-correlation functionals [28] and shows that this kind of Ta-Ni hybridization cannot spontaneously form due to exciton condensation below a critical temperature $[26,27,38]$.

In contrast to that, we will show below that the excitonic instability can break the crystal symmetries that constrain Ta-Ni hybridization in the high-temperature phase. In particular, the reflection symmetries $\sigma_{\perp}^{A / B}$ constrain the Ta-Ni hoppings to change sign under $\sigma_{\perp}^{A / B}$ implying that the Wannier Hamiltonian averaged along the $x$ direction is block diagonal with respect to the Ta and Ni states

$\hat{H}\left(k_{x}=0, R_{y}\right) \equiv \sum_{R_{x}} \hat{H}\left(R_{x}, R_{y}\right)=\left(\begin{array}{cc}\hat{h}_{\mathrm{Ta}}\left(R_{y}\right) & \hat{0} \\ \hat{0} & \hat{h}_{\mathrm{Ni}}\left(R_{y}\right)\end{array}\right)$.

In momentum space, $t_{\mathrm{TaNi}}\left(k_{x}=0, k_{y}\right)=0$ so that the bands along the $\overline{Z \Gamma}$ path have purely $\mathrm{Ta}$ or Ni character, Fig. 2. Therefore, any excitonic instability resulting from a spontaneous Ta-Ni hybridization must break the $\boldsymbol{\sigma}_{\perp}^{A / B}$ symmetry and show up along the $\overline{Z \Gamma}$ direction. We provide evidence of such an instability by considering the effect of the electron interactions in a minimal model derived from the above symmetry analysis.

Minimal model.-We consider a two-dimensional lattice with six atoms per unit cell. The electronic Hamiltonian

$$
\hat{\mathcal{H}}=\hat{H}_{\text {hop }}+\hat{H}_{U}+\hat{H}_{V}
$$

includes a hopping term $\hat{H}_{\text {hop }}$, a local $\hat{H}_{U}$ Coulomb interaction term, and nearest-neighbor $\hat{H}_{V}$ one. We define $\Psi_{\vec{R} \sigma}^{\dagger} \equiv\left(c_{1 \sigma}^{\dagger}(\vec{R}), \ldots, c_{6 \sigma}^{\dagger}(\vec{R})\right)$, where $c_{j \sigma}^{\dagger}(\vec{R})$ creates an electron with spin $\sigma$ in a localized orbital on the $j$ th atom (labels in Fig. 1) of the unit cell $\vec{R}$.

$$
\hat{H}_{\text {hop }}=\sum_{\vec{R} \sigma} \sum_{\vec{\delta}} \Psi_{\vec{R}+\vec{\delta} \sigma}^{\dagger} \mathbf{T}(\vec{\delta}) \Psi_{\vec{R} \sigma}
$$

contains intracell, $\mathbf{T}(\overrightarrow{0})$, as well as intercell, $\mathbf{T}( \pm a, \pm b)$, terms. The matrix elements are chosen consistently with the above symmetry requirement and in order to reproduce the main features of the Wannier band structure [28]. (a) Symmetry invariant

(b) Symmetry broken
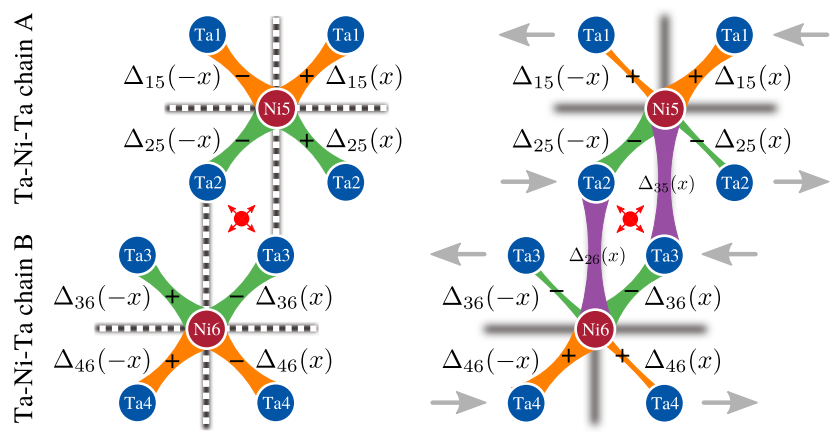

FIG. 3. Scheme of nearest neighbor Ta-Ni hybridization in the symmetry invariant (a) and symmetry-broken (b) phases. Thickness of the lines connecting the atoms indicates the absolute value of the hybridization.

The electrons interact through a local Hubbard-like term

$$
\hat{H}_{U}=U \sum_{\vec{R}} \sum_{j} \hat{n}_{j \uparrow}(\vec{R}) \hat{n}_{j \downarrow}(\vec{R})
$$

where we assumed the same $U$ for the six atoms, as supported by a constrained RPA [39] analysis of the Coulomb matrix elements $\left(U_{\mathrm{Ta}} \approx 2.1 \mathrm{eV}, U_{\mathrm{Ni}} \approx 2.4 \mathrm{eV}\right)$. The next leading terms are intrachain density-density interactions between neighboring $\mathrm{Ta}$ and $\mathrm{Ni}$ atoms $(V \approx 0.9 \mathrm{eV})$

$$
\begin{aligned}
\hat{H}_{V}= & V \sum_{j=1,2} \sum_{\vec{R} \sigma \sigma^{\prime}}\left[\hat{n}_{j \sigma}(\vec{R})+\hat{n}_{j \sigma}\left(\vec{R}+\vec{\delta}_{x}\right)\right] \hat{n}_{5 \sigma^{\prime}}(\vec{R}) \\
& +V \sum_{j=3,4} \sum_{\vec{R} \sigma \sigma^{\prime}}\left[\hat{n}_{j \sigma}(\vec{R})+\hat{n}_{j \sigma}\left(\vec{R}-\vec{\delta}_{x}\right)\right] \hat{n}_{6 \sigma^{\prime}}(\vec{R}) .
\end{aligned}
$$

The symmetries of the Hamiltonian are revealed by an investigation of the intrachain Ta-Ni hybridization as a function of the distance along $x$ between the $\mathrm{Ta}$ and $\mathrm{Ni}$ atoms

$$
\Delta_{i j}(x)=\langle\underbrace{c_{i}^{\dagger}\left(R_{x}, 0\right)}_{\text {Ta site }} \underbrace{c_{j}(0,0)}_{\text {Ni site }}\rangle,
$$

where $i=1,2(3,4)$ and $j=5(6)$ label the $\mathrm{Ta}$ and $\mathrm{Ni}$ states, respectively, for the $A(B)$ chain. For each chain, $x$ is defined by taking the $\mathrm{Ni}$ atom in that chain as origin, so that $x=R_{x} \mp a / 2(-$ for $A$ and + for $B)$. We have dropped the spin index as we focus on the spin-singlet case. For the $A$ chain, $\Delta_{15}(x)$ and $\Delta_{25}(x)$, i.e., the hybridizations between the lower and upper Ta with the central $\mathrm{Ni}$ state of the $A$ chain, transform as

$$
\sigma_{\perp}^{A} \Delta_{15}(x)=-\Delta_{15}(-x), \quad \sigma_{\|}^{A} \Delta_{15}(x)=\Delta_{25}(x),
$$

so that $\Delta_{15}(x)=-\Delta_{15}(-x)$ and $\Delta_{15}(x)=\Delta_{25}(x)$, as depicted in Fig. 3(a). Similarly, reflection symmetries for 

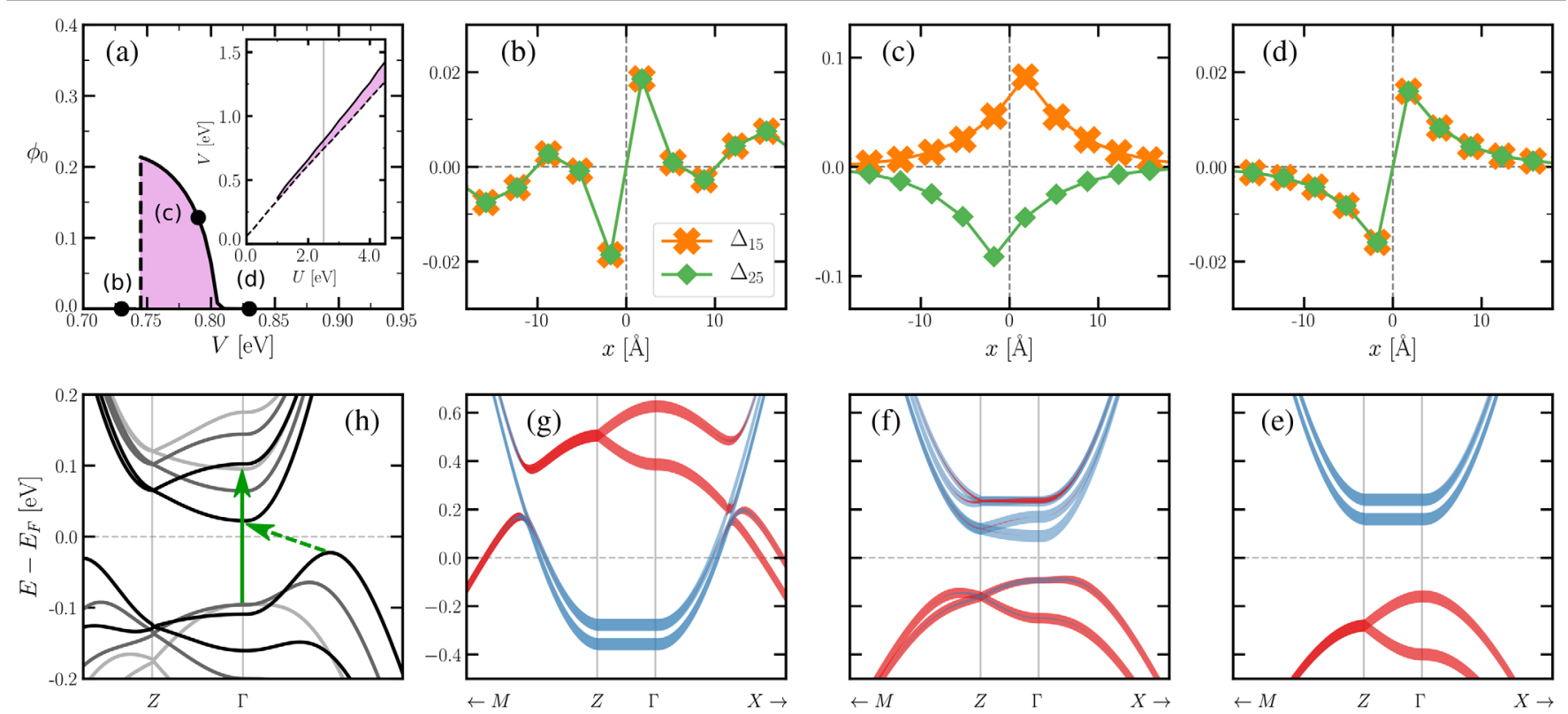

FIG. 4. (a) Order parameter at zero temperature as function of $V$ for $U=2.50 \mathrm{eV}$. Dots represent $V=0.73, V=0.785$, and $V=0.83$ corresponding to panels (b)-(d). Inset: phase diagram in $U-V$ plane. Shaded region corresponds to the symmetry-broken phase. Dashed line indicates a metal-insulator Lifshitz transition. (b)-(d) Ta-Ni hybridization along Ta-Ni-Ta A chain in the symmetric (b) and (d) and symmetry-broken (c) phases. Crosses (diamonds) correspond to upper (lower) part of the Ta-Ni-Ta chain. (e)-(g) Bands along $M-$ $Z-\Gamma-X$ corresponding to panels (b) $-(\mathrm{d})$. Red (blue) corresponds to Ni (Ta) character. (h) Gap evolution inside the broken-symmetry phase. $U=2.50 \mathrm{eV}$ and $V=0.79,0.77,0.75 \mathrm{eV}$ from light grey to black lines. Full (dashed) green arrows highlight the direct (indirect) gap.

the $B$ chains imply $\Delta_{36}(x)=-\Delta_{36}(-x)$ and $\Delta_{46}(x)=$ $-\Delta_{46}(-x)$.

We investigate the possible breaking of the above symmetries due to electronic interactions, by utilizing a Hartree-Fock variational wave function allowing for a spatially homogeneous order parameter of the form:

$$
\vec{\phi}=\left(\begin{array}{c}
\phi_{15} \\
\phi_{25} \\
\phi_{36} \\
\phi_{46}
\end{array}\right), \quad \phi_{i j} \equiv \Delta_{i j}(a / 2)+\Delta_{i j}(-a / 2)
$$

The four $\phi_{i j}$ are in general independent, allowing in principle for 16 different phases corresponding to the different breaking patterns of the reflections and inversion symmetries. Here, we focus on the symmetry-breaking channel consistent with the low-temperature monoclinic phase of $\mathrm{Ta}_{2} \mathrm{NiSe}_{5}$. In the monoclinic phase all reflections are broken, while their products $I^{A / B}=\sigma_{\perp}^{A / B} \sigma_{\|}^{A / B}$ and the inversion $\mathcal{I}=I^{A / B} \mathcal{T}(\mathcal{T}$ being the translation between the two Ni atoms) are preserved. This constrains the components $\phi_{i j}$ as $\phi_{15}=-\phi_{25}$ and $\phi_{36}=-\phi_{46}$, due to preservation of $I^{A / B}$ and $\phi_{15}=\phi_{46}$ and $\phi_{25}=\phi_{36}$ due to $\mathcal{I}$, leading to an order parameter of the form $\vec{\phi}=\phi_{0}(+1,-1,-1,+1)$.

The obtained zero-temperature phase diagram in the $U-V$ plane, Fig. 4(a), shows three distinct regions. At fixed value of $U$, the order parameter $\phi_{0}$ vanishes for $V$ smaller than a lower critical value $\left[V<V_{l}^{*}(U)\right]$ and for $V$ larger than an upper critical value $\left[V>V_{u}^{*}(U)\right]$. In these regions the Ta-Ni hybridizations transform in accordance with Eq. (7), as shown in Figs. 4(b) and 4(d). These two symmetric ground states are characterized by different electronic properties. For $V<V_{l}^{*}(U)$ [Figs. 4(b) and 4(g)] the valence and conduction bands overlap, while for $V>V_{u}^{*}(U)$ [Figs. 4(d) and 4(e)] the bands are separated by an energy gap.

In the intermediate region $V_{l}^{*}(U)<V<V_{u}^{*}(U)$ [Figs. 4(c) and 4(f)] a solution with $\phi_{0} \neq 0$ is stabilized. This is the hallmark of the excitonic instability as witnessed in Fig. 4(f) by the emergence of a sizable hybridization between valence and conduction bands all along the $\overline{Z \Gamma}$ path. Valence and conduction bands acquire a strong $\mathrm{Ta}$ and Ni character, respectively (which is absent in the symmetric phase) and the degeneracy of the Ta-like conduction bands along $\overline{Z \Gamma}$ is lifted by hybridization with $\mathrm{Ni}$-like valence bands. In real space this translates into broken $\sigma_{\perp}^{A / B}$ and $\sigma_{\|}^{A / B}$ symmetries yielding finite $\Delta_{35}$ and $\Delta_{26}$, which couple the two chains, Fig. 3(b).

The upper valence band develops a mostly flat dispersion around $\Gamma$. While this has so far been interpreted as a distinctive signature of an homogeneous excitonic condensate, we show here that the interpretation is not unique. In fact this feature is a result of a direct-to-indirect gap insulator transition, driven by the splitting between the hybridized bands along $\overline{Z \Gamma}$, that can occur inside the 
broken-symmetry phase [Fig. 4(h)]. By decreasing $V$ the splitting increases, while the bottom of the conduction band moves closer to the Fermi level. At $V=V_{l}^{*}(U)$ the conduction band crosses the Fermi level and the system undergoes a Lifshitz transition accompanied by the formation of a Fermi surface in the metallic phase. We find here that this is a first-order transition which restores the symmetry for $V<V_{l}^{*}(U)$. If we allowed for a nonhomogeneous $\vec{\phi}(\vec{R})$ order parameter a finite momentum instability could occur near this point [40]. The symmetrybroken phase is found only in a small region of the phase space close to the Lifshitz transition. In this regime the symmetric phase is characterized by a very small gap, reinforcing the relevance of the above phase transition for $\mathrm{Ta}_{2} \mathrm{NiSe}_{5}$, which in the high-temperature phase has been reported to be a zero-gap semiconductor [16]. The symmetry-broken region shrinks as the Hubbard $U$ is decreased until it disappears for $U \lesssim 1.25 \mathrm{eV}$ for which $V_{l}^{*}(U)$ and $V_{u}^{*}(U)$ merge into the Lifshitz transition line. We highlight that our constrained RPA values for $U$ and $V$ are in close vicinity of the symmetry-broken region.

Structural phase transition.-The electronic configuration associated with the excitonic phase is not compatible with the symmetries of the lattice. This implies that the electronic order parameter must have a linear coupling to lattice modes breaking the crystal symmetry [27,41]. Hence, the excitonic transition will coexist with a structural transition, as indeed observed experimentally. From the pattern of the Ta-Ni hybridizations in the broken-symmetry phase [Fig. 3(b)], one anticipates a distortion of the unit cell in which Ta atoms from the same Ta-Ni-Ta chain are tilted in opposite directions [arrows in Fig. 3(b)]. This corresponds to a structural transition from the orthorhombic to the monoclinic structure, which we confirm to be present by performing a full structural relaxation within DFT [28]. The interplay between the electronic and lattice instability is an interesting question for future investigations [42].

Conclusions. - We have performed a symmetry analysis backed up by first-principle calculations of $\mathrm{Ta}_{2} \mathrm{NiSe}_{5}$, with general implications for the excitonic transitions in solids. While this transition has been so far understood as a condensation phenomenon resulting from a continuous $U_{X}(1)$ symmetry breaking [27] we show that in realistic solids there is no such symmetry: the purely electronic transition corresponds to the breaking of discrete symmetries only. Important consequences include that all collective modes are gapped and that there is no dissipationless transport or excitonic superfluidity. We identify explicitly all discrete symmetries relevant for the structural phase transition in $\mathrm{Ta}_{2} \mathrm{NiSe}_{5}$, including the corresponding electronic order parameters and provide clear evidence for a transition into an excitonic insulator phase. This transition breaks these symmetries in a manner consistent with the experimentally observed lattice distortion into a monoclinic phase, and the order parameter couples linearly to lattice modes.

Because we find a spontaneous electronic instability for realistic values of the interactions, our results suggest an electronic contribution to the coupled transition [43-45]. However, a definitive confirmation of this point calls for experimental probes which can selectively address the electronic and lattice degrees of freedom. In the context of iron-based superconductors, where a similar question arises in relation to nematicity, it has proven possible to probe the electronic component of the susceptibility associated with the nematic instability $[46,47]$. Ultrafast spectroscopies offer another possible route $[22,25,48,49]$ by exploiting the very different time scales associated with electronic and lattice degrees of freedom.

Discussions with Jennifer Cano, Denis Golež, Edoardo Baldini, Selene Mor, Tatsuya Kaneko, and Jernej Mravlje are gratefully acknowledged. We thank Merzuk Kaltak for sharing his (c)RPA implementation [50] with us. This work was supported by (A. G., G. M.) the European Research Council (ERC-319286-QMAC) and (A. M.) the US Department of Energy under Grant No. DE-SC 0019443. G. M. acknowledges support from the Swiss National Science Foundation Ambizione Grant No. PZ00P2_186146. S. L., L. W., H. H., and A. R. were supported by the European Research Council (Grant No. ERC-2015-AdG694097), the Cluster of Excellence AIM, Grupos Consolidados (Grant No. IT1249-19) and SFB925. S. L. acknowledges support from the Alexander von Humboldt foundation. The Flatiron Institute is a division of the Simons Foundation.

\footnotetext{
*These two authors equally contributed.

†giacomo.mazza@unige.ch

¥m.roesner@science.ru.nl

§ageorges@flatironinstitute.org
}

[1] A. N. Kozlov and L. A. Maksimov, Sov. J. Exp. Theor. Phys. 21, 790 (1965).

[2] L. V. Keldysh and A. N. Kozlov, Sov. J. Exp. Theor. Phys. 27, 521 (1968).

[3] D. Jérome, T. M. Rice, and W. Kohn, Phys. Rev. 158, 462 (1967).

[4] B. I. Halperin and T. M. Rice, Rev. Mod. Phys. 40, 755 (1968).

[5] J. Eisenstein, Annu. Rev. Condens. Matter Phys. 5, 159 (2014).

[6] M. Kellogg, J. P. Eisenstein, L. N. Pfeiffer, and K. W. West, Phys. Rev. Lett. 93, 036801 (2004).

[7] I. B. Spielman, J. P. Eisenstein, L. N. Pfeiffer, and K. W. West, Phys. Rev. Lett. 84, 5808 (2000).

[8] J. I. A. Li, T. Taniguchi, K. Watanabe, J. Hone, and C. R. Dean, Nat. Phys. 13, 751 (2017).

[9] X. Liu, K. Watanabe, T. Taniguchi, B. I. Halperin, and P. Kim, Nat. Phys. 13, 746 (2017).

[10] D. Snoke, Science 298, 1368 (2002). 
[11] L. V. Butov, C. W. Lai, A. L. Ivanov, A. C. Gossard, and D. S. Chemla, Nature (London) 417, 47 (2002).

[12] L. V. Butov, A. C. Gossard, and D.S. Chemla, Nature (London) 418, 751 (2002).

[13] F. D. Salvo, C. Chen, R. Fleming, J. Waszczak, R. Dunn, S. Sunshine, and J. A. Ibers, J. Less Common Metals 116, 51 (1986).

[14] Y. Wakisaka, T. Sudayama, K. Takubo, T. Mizokawa, M. Arita, H. Namatame, M. Taniguchi, N. Katayama, M. Nohara, and H. Takagi, Phys. Rev. Lett. 103, 026402 (2009).

[15] K. Seki, Y. Wakisaka, T. Kaneko, T. Toriyama, T. Konishi, T. Sudayama, N. L. Saini, M. Arita, H. Namatame, M. Taniguchi, N. Katayama, M. Nohara, H. Takagi, T. Mizokawa, and Y. Ohta, Phys. Rev. B 90, 155116 (2014).

[16] Y. F. Lu, H. Kono, T. I. Larkin, A. W. Rost, T. Takayama, A. V. Boris, B. Keimer, and H. Takagi, Nat. Commun. 8, 14408 (2017).

[17] F. J. Di Salvo, D. E. Moncton, and J. V. Waszczak, Phys. Rev. B 14, 4321 (1976).

[18] H. Cercellier, C. Monney, F. Clerc, C. Battaglia, L. Despont, M. G. Garnier, H. Beck, P. Aebi, L. Patthey, H. Berger, and L. Forró, Phys. Rev. Lett. 99, 146403 (2007).

[19] A. Kogar, M. S. Rak, S. Vig, A. A. Husain, F. Flicker, Y. I. Joe, L. Venema, G. J. MacDougall, T. C. Chiang, E. Fradkin, J. van Wezel, and P. Abbamonte, Science 358, 1314 (2017).

[20] A. Nakano, T. Hasegawa, S. Tamura, N. Katayama, S. Tsutsui, and H. Sawa, Phys. Rev. B 98, 045139 (2018).

[21] S. Y. Kim, Y. Kim, C.-J. Kang, E.-S. An, H. K. Kim, M. J. Eom, M. Lee, C. Park, T.-H. Kim, H. C. Choi, B. I. Min, and J. S. Kim, ACS Nano 10, 8888 (2016).

[22] S. Mor, M. Herzog, D. Golež, P. Werner, M. Eckstein, N. Katayama, M. Nohara, H. Takagi, T. Mizokawa, C. Monney, and J. Stähler, Phys. Rev. Lett. 119, 086401 (2017).

[23] J. Lee, C.-J. Kang, M. J. Eom, J. S. Kim, B. I. Min, and H. W. Yeom, Phys. Rev. B 99, 075408 (2019).

[24] T. I. Larkin, A. N. Yaresko, D. Pröpper, K. A. Kikoin, Y. F. Lu, T. Takayama, Y.-L. Mathis, A. W. Rost, H. Takagi, B. Keimer, and A. V. Boris, Phys. Rev. B 95, 195144 (2017).

[25] D. Werdehausen, T. Takayama, M. Höppner, G. Albrecht, A. W. Rost, Y. Lu, D. Manske, H. Takagi, and S. Kaiser, Sci. Adv. 4, eaap8652 (2018).

[26] K. Sugimoto, S. Nishimoto, T. Kaneko, and Y. Ohta, Phys. Rev. Lett. 120, 247602 (2018).

[27] T. Kaneko, T. Toriyama, T. Konishi, and Y. Ohta, Phys. Rev. B 87, 035121 (2013).

[28] See the Supplemental Material at http://link.aps.org/ supplemental/10.1103/PhysRevLett.124.197601 containing details on the $a b$ initio calculations and the construction of the model Hamiltonian, which includes Refs. [29-37].

[29] J. P. Perdew, K. Burke, and M. Ernzerhof, Phys. Rev. Lett. 77, 3865 (1996).

[30] P. E. Blöchl, Phys. Rev. B 50, 17953 (1994).

[31] G. Kresse and J. Furthmüller, Comput. Mater. Sci. 6, 15 (1996).

[32] G. Kresse and J. Furthmüller, Phys. Rev. B 54, 11169 (1996).

[33] S. A. Sunshine and J. A. Ibers, Inorg. Chem. 24, 3611 (1985).

[34] A. D. Becke and E. R. Johnson, J. Chem. Phys. 124, 221101 (2006).

[35] F. Tran and P. Blaha, Phys. Rev. Lett. 102, 226401 (2009).

[36] A. A. Mostofi, J. R. Yates, Y.-S. Lee, I. Souza, D. Vanderbilt, and N. Marzari, Comput. Phys. Commun. 178, 685 (2008).

[37] E. Sasioglu, C. Friedrich, and S. Blügel, Phys. Rev. B 83, 121101(R) (2011).

[38] S. Ejima, T. Kaneko, Y. Ohta, and H. Fehske, Phys. Rev. Lett. 112, 026401 (2014).

[39] F. Aryasetiawan, M. Imada, A. Georges, G. Kotliar, S. Biermann, and A. I. Lichtenstein, Phys. Rev. B 70, 195104 (2004).

[40] K. Domon, T. Yamada, and Y. Ono, J. Phys. Soc. Jpn. 87, 054701 (2018).

[41] B. Zenker, H. Fehske, and H. Beck, Phys. Rev. B 90, 195118 (2014).

[42] L. Windgätter, M. Rösner, S. Latini, G. Mazza, H. Hübener, A. J. Millis, A. Rubio, and A. Georges (to be published).

[43] M. D. Watson, I. Marković, E. A. Morales, P. L. Fèvre, M. Merz, A. A. Haghighirad, and P. D. C. King, Phys. Rev. Research 2, 013236 (2020).

[44] A. Subedi, arXiv:2002.08352.

[45] T. Tang, H. Wang, S. Duan, Y. Yang, C. Huang, Y. Guo, D. Qian, and W. Zhang, arXiv:2003.00514.

[46] J.-H. Chu, H.-H. Kuo, J. G. Analytis, and I. R. Fisher, Science 337, 710 (2012).

[47] P. Massat, D. Farina, I. Paul, S. Karlsson, P. Strobel, P. Toulemonde, M.-A. Méasson, M. Cazayous, A. Sacuto, S. Kasahara, T. Shibauchi, Y. Matsuda, and Y. Gallais, Proc. Natl. Acad. Sci. U.S.A. 113, 9177 (2016).

[48] Y. Murakami, D. Golež, M. Eckstein, and P. Werner, Phys. Rev. Lett. 119, 247601 (2017).

[49] M. Porer, U. Leierseder, J.-M. Menard, H. Dachraoui, L. Mouchliadis, I. E. Perakis, U. Heinzmann, J. Demsar, K. Rossnagel, and R. Huber, Nat. Mater. 13, 857 (2014).

[50] M. Kaltak, Merging GW with DMFT, Ph.D. Thesis, University of Vienna, 2015. 